\title{
The Therapeutic Role of Nanoparticle Shape in Traumatic Brain Injury : An in vitro Comparative Study
}

\author{
Dong Hyuk Youn, ${ }^{1, *}$ Harry Jung, ${ }^{1, *}$ Ngoc Minh Tran, ${ }^{2}$ Jin Pyeong Jeon, ${ }^{1,3}$ Hyojong Yoo ${ }^{2}$ \\ Institute of New Frontier Research,' Hallym University College of Medicine, Chuncheon, Korea \\ Department of Materials Science and Chemical Engineering, ${ }^{2}$ Hanyang University, Ansan, Korea \\ Department of Neurosurgery, Hallym University College of Medicine, Chuncheon, Korea
}

Objective : To perform a comparative analysis of therapeutic effects associated with two different shapes of ceria nanoparticles, ceria nanorods (Ceria NRs) and ceria nanospheres (Ceria NSs), in an in vitro model of traumatic brain injury (TBI).

Methods : In vitro TBI was induced using six-well confluent plates by manually scratching with a sterile pipette tip in a $6 \times 6$-square grid. The cells were then incubated and classified into cells with scratch injury without nanoparticles and cells with scratch injury, which were treated separately with $1.16 \mathrm{mM}$ of Ceria NSs and Ceria NRs. Antioxidant activities and anti-inflammatory effects were analyzed.

Results : Ceria NRs and Ceria NSs significantly reduced the level of reactive oxygen species compared with the control group of SH-SY5Y cells treated with Dulbecco's phosphate-buffered saline. The mRNA expression of superoxide dismutases was also reduced in nanoparticle-treated SH-SY5Y cells, but apparently the degree of mRNA expression decrease was not dependent on the nanoparticle shape. Exposure to ceria nanoparticles also decreased the cyclooxygenase-2 expression, especially prominent in Ceria NR-treated group than that in Ceria NS-treated group.

Conclusion : Ceria nanoparticles exhibit antioxidant and anti-inflammatory effects in TBI models in vitro. Ceria NRs had better antiinflammatory effect than Ceria NSs, but showed similar antioxidant activity.

Key Words : Traumatic brain injury · Cerium oxide $\cdot$ Nanoparticles.

\section{INTRODUCTION}

Traumatic brain injury (TBI) induces brain damage via primary and secondary injuries ${ }^{13)}$. Primary brain injury entails compression, displacement, stretching, shearing, and tearing of the brain parenchyma and cerebral blood vessels. In con- trast, secondary injuries occur over hours and days after trauma, and result in brain damage mediated via a series of complex biochemical cascades including oxidative stress due to accumulation of reactive oxygen species (ROS), neuroinflammation, calcium-dependent excitotoxicity, and mitochondrial dysfunction $^{8)}$. These secondary injuries can lead to structural

- Received : July 21, 2021 •Revised : September 16, 2021 •Accepted : September 23, 2021

- Address for reprints : Jin Pyeong Jeon

Department of Neurosurgery, Hallym University College of Medicine, 77 Sakju-ro, Chuncheon 24253, Korea

Tel : +82-33-240-5171, Fax : +82-33-240-9970, E-mail : jjs6553@daum.net, ORCID : https://orcid.org/0000-0001-8543-6855

*Dong Hyuk Youn and Harry Jung contributed equally to this work and should be considered co-first authors.

This is an Open Access article distributed under the terms of the Creative Commons Attribution Non-Commercial License (http://creativecommons.org/licenses/by-nc/4.0) which permits unrestricted non-commercial use, distribution, and reproduction in any medium, provided the original work is properly cited. 
changes and neurodegeneration of the brain, in spite of adequate surgical or medical management of the primary injury. Accordingly, efforts have been made to reduce secondary brain damage; however, a clinically effective drug has yet to discovered.

Currently, several studies are investigating the effects of cerium oxides (ceria nanoparticles) as therapeutic agents. Oxygen storage mediated by changes in surface $\mathrm{Ce}^{4+}$ and $\mathrm{Ce}^{3+}$ provides catalytic activity during the redox reaction. Ceria nanoparticles exhibit higher catalytic activity than superoxide dismutase (SOD) and catalase enzymes ${ }^{15,21)}$. Also, the radical scavenging activity is regenerated and sustained in the biological process $^{2,18)}$. However, nanoparticle-based therapeutic studies in the field of TBI are limited compared with cancer or neurodegenerative diseases involving the brain. Bailey et al. ${ }^{2)}$ reported that spherical ceria nanoparticles (Ceria NSs) improved cognitive function via reduced neuronal death and calcium dysregulation in a rodent model of mild TBI. Theoretically, the varying morphology determines the composition of $\mathrm{Ce}^{4+}$ and $\mathrm{Ce}^{3+}$ on the surface, which alters the catalytic activity. Tube-shaped ce- ria nanoparticles exhibit larger Brunauer-Emmett-Teller surface area than the other types of ceria nanoparticles. Ceria nanorods (Ceria NRs) showed a higher catalytic effect due the exposed crystal surface (110) of ceria compared with nonocubes, nanotubes or nanowires ${ }^{16)}$. Therefore, it is necessary to analyze the therapeutic effects of nanoparticle shape on the TBI. Here, we compared the therapeutic effect of Ceria NRs and Ceria NSs in an in vitro model of TBI to identify a clinically effective ceria agent.

\section{MATERIALS AND METHODS}

All experiments were approved by the Institutional Animal Care and Use Committee of the Hallym University (approval No. R2 2019-35).

\section{Synthesis of ceria nanoparticles}

Ceria NRs were synthesized using a previously reported protocol with a slight modification (Fig. 1A) ${ }^{19)}$. Cerium nitrate
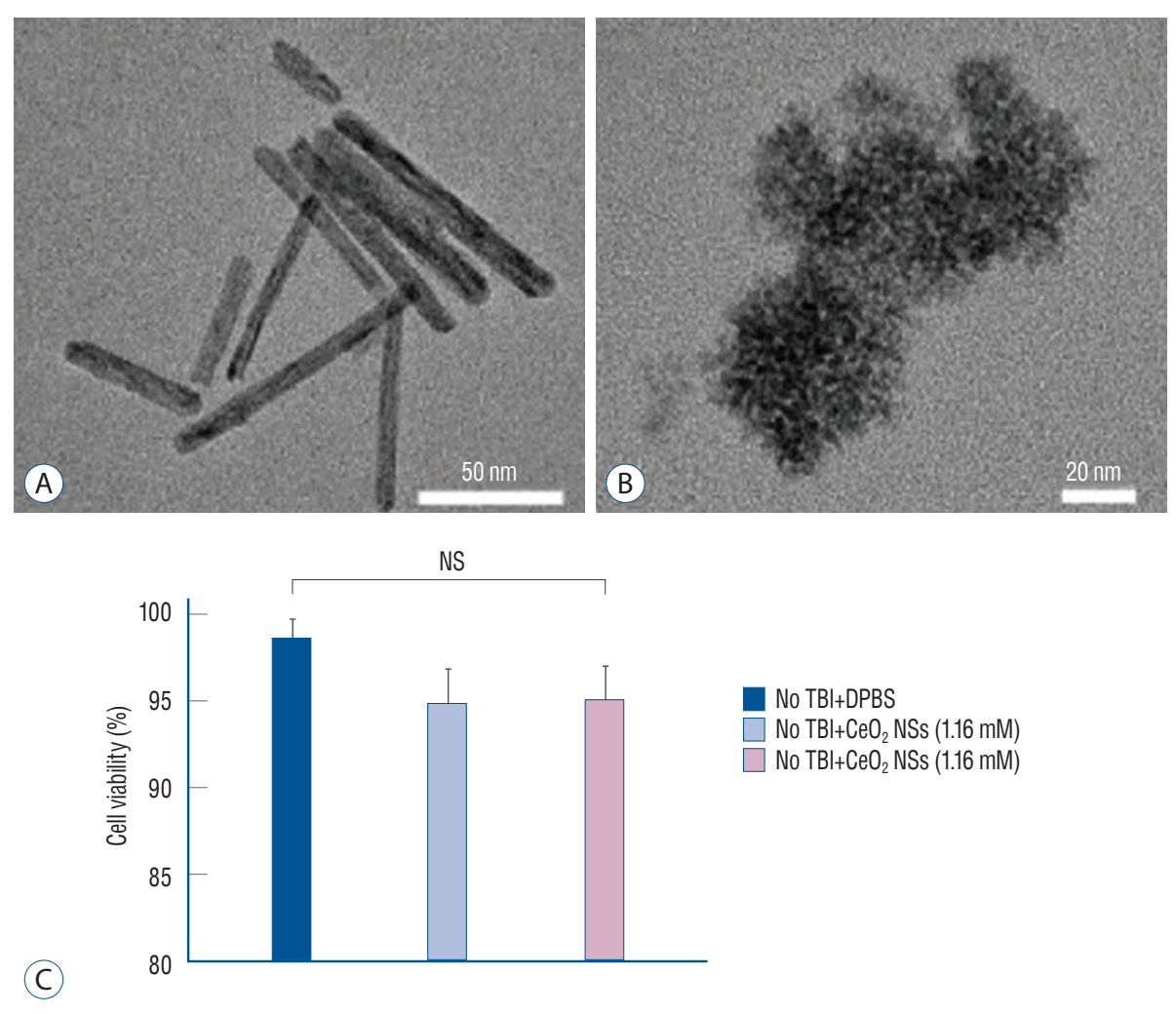

Fig. 1. Transmission electron microscopic images of ceria nanorods (Ceria NRs) (A) and ceria nanospheres (Ceria NSs) (B). 3-(4,5-dimethylthiazol-2-YI)2,5-diphenyltetrazolium bromide (MTT) cell viability assay of SH-SY5Y cells treated with ceria nanoparticles (C). NS : non-specific, TBI : traumatic brain injury, DPBS : Dulbecco's phosphate-buffered saline. 
hexahydrate $(0.434 \mathrm{~g}, 1 \mathrm{mmol})$ was added to an aqueous solution of sodium hydroxide $(10 \mathrm{M}, 20 \mathrm{~mL})$ in a PTFE beaker. The reaction mixture was vigorously stirred for 2 hours at room temperature before transfer to a Teflon-lined stainlesssteel autoclave, followed by incubation in a temperature-controlled oven at $100^{\circ} \mathrm{C}$ for 10 hours. The mixture was naturally cooled to room temperature after completion of the reaction. The resultant product was centrifuged, followed by washing repeatedly with solvents, and then vacuum-dried for 24 hours. Ceria NSs were also synthesized according to a previous protocol with a slight modification (Fig. 1B) $)^{10)}$. Cerium nitrate hexahydrate $(0.434 \mathrm{~g}, 1 \mathrm{mmol})$ and oleylamine $(3.25 \mathrm{~g}, 12$ $\mathrm{mmol})$ were dissolved in xylene $(15 \mathrm{~mL})$. The resulting solution was vigorously stirred for 2 hours at $25^{\circ} \mathrm{C}$ and then heated to $90^{\circ} \mathrm{C}$ at the rate of $2^{\circ} \mathrm{C} \cdot \mathrm{min}^{-1}$ under vacuum. Deionized water $(1 \mathrm{~mL})$ was rapidly injected into the solution under vigorous stirring at $90^{\circ} \mathrm{C}$ to initiate the sol-gel reaction until the color changed from purple to cloudy yellow. The reaction mixture was incubated at $90^{\circ} \mathrm{C}$ for 3 hours to obtain a lightyellow colloidal solution. The mixture was naturally cooled to ambient temperature, and Ceria NSs were precipitated by adding ethanol $(75 \mathrm{~mL})$. The obtained product was collected by centrifugation and washed repeatedly with ethanol, and then dried under vacuum for 24 hours for further use ${ }^{20)}$.

\section{Cell culture and culture conditions}

Human histiocytic lymphoma (U-937), human neuroblastoma (SH-SY5Y), and mouse normal monocyte macrophage
(RAW 264.7) were used for in vitro experiments. U-937 cells were maintained in Roswell Park Memorial Institute medium (Welgene Inc., Daegu, Korea) 1640 supplemented with 10\% heat-inactivated fetal bovine serum (FBS; Welgene Inc.) and $1 \%$ penicillin streptomycin (P/S; Gibco, Brooklyn, NY, USA). The SH-SY5Y cells were cultured in Ham's-F12/Minimum Essential medium (1 : 1) with 1\% P/S. The RAW 264.7 cells were cultured in Dulbecco's modified Eagle's medium (Welgene Inc.) containing 10\% FBS and 1\% P/S. All cell lines were maintained at $37^{\circ} \mathrm{C}$ in a humidified atmosphere of $5 \% \mathrm{CO}_{2}$ for optimal growth.

\section{Comparison of ceria nanoparticles}

Antioxidant and anti-inflammatory effects of Ceria NRs and Ceria NSs were investigated and compared (Fig. 2). The superoxide anion scavenging activity was assessed in vitro using a Superoxide Anion Assay Kit (CS1000-1KT; SigmaAldrich, Saint Louis, MO, USA). U-937 cells were cultured for 48 hours and activated by treatment with $1 \mu \mathrm{g} / \mathrm{mL}$ of lipopolysaccharides (LPS; L2630; Sigma, Saint Louis, MO, USA) for 24 hours. A U-937 cell suspension of $5.0 \times 10^{5}$ cells was added to each well. The reaction was initiated by adding $20 \mu \mathrm{g} / \mathrm{mL}$ of phorbol-12-myristate-13-acetate (PMA; P8139; Sigma), luminol solution, enhancer solution, and assay buffer according to the kit manufacturer's protocol. A buffer containing $1.16 \mathrm{mM}$ of Ceria NSs and Ceria NRs was used at the time of assay. The intensity of superoxide anion-induced luminescence intensity was measured every 10 minutes for 4 hours. Nitric oxide (NO)
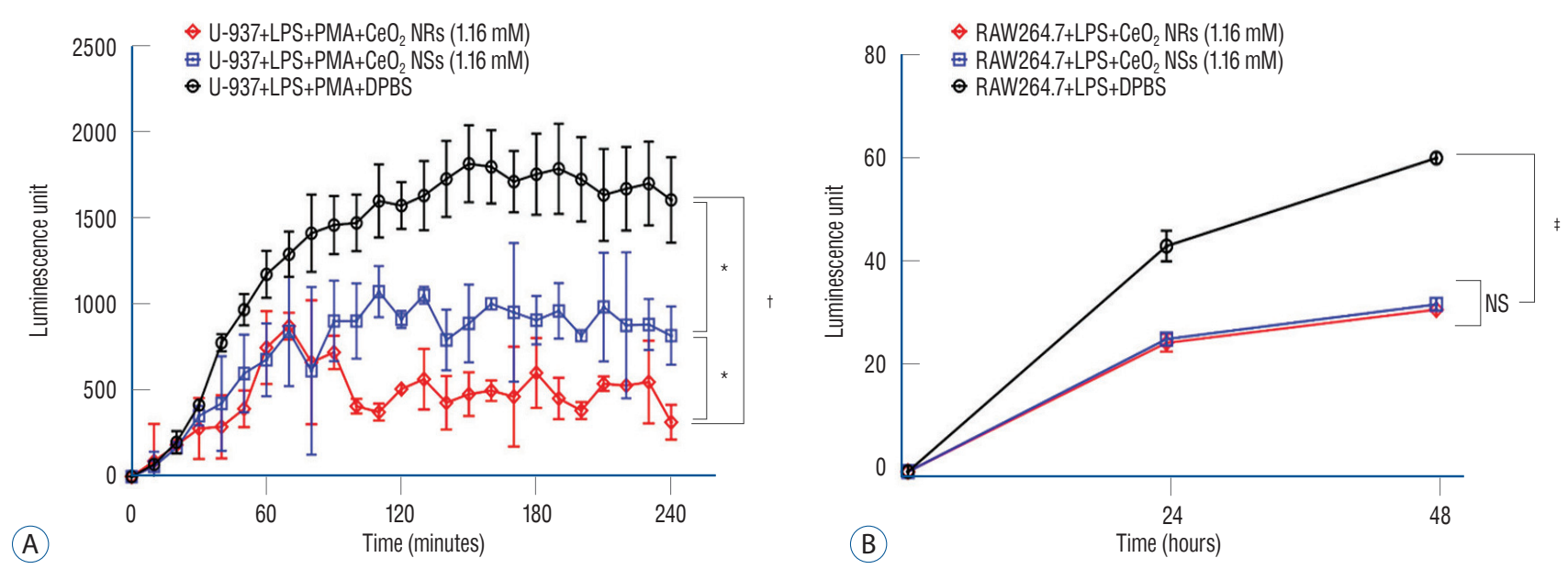

Fig. 2. Comparison of antioxidant (A) and anti-inflammatory effects (B) of ceria nanorods (Ceria NRs) and ceria nanospheres (Ceria NSs). Assay for the measurement of superoxide anions and nitric oxide (NO) levels in LPS-induced inflammation were performed separately. ${ }^{*} p<0.05 .{ }^{\dagger} p<0.01 .{ }^{\ddagger} p<0.001$. LPS : lipopolysaccharides, PMA : phorbol-12-myristate-13-acetate, DPBS : Dulbecco's phosphate-buffered saline, NS : non-specific. 
(A)
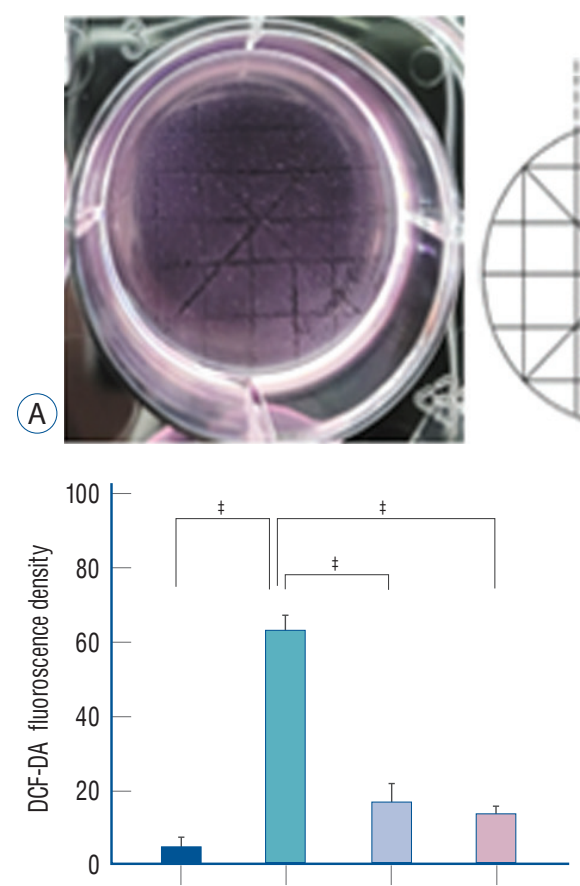

(C)

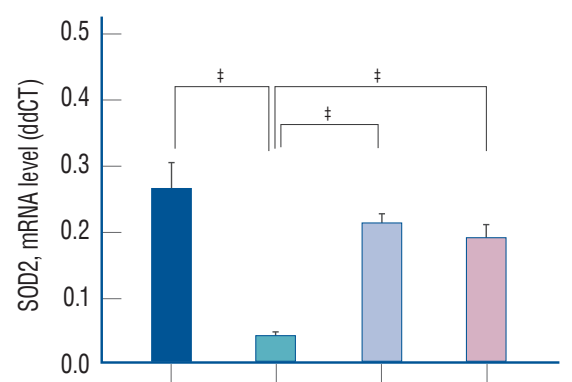

(E)

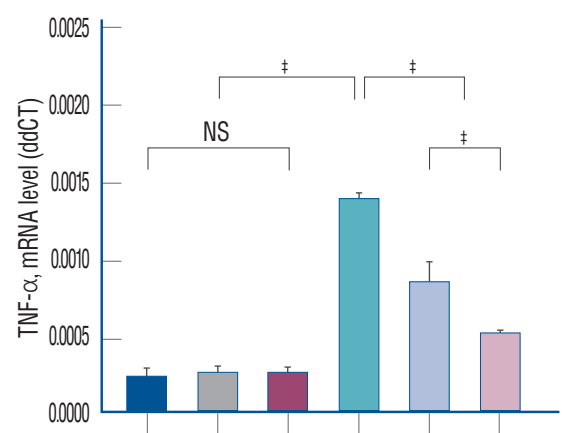

(H)
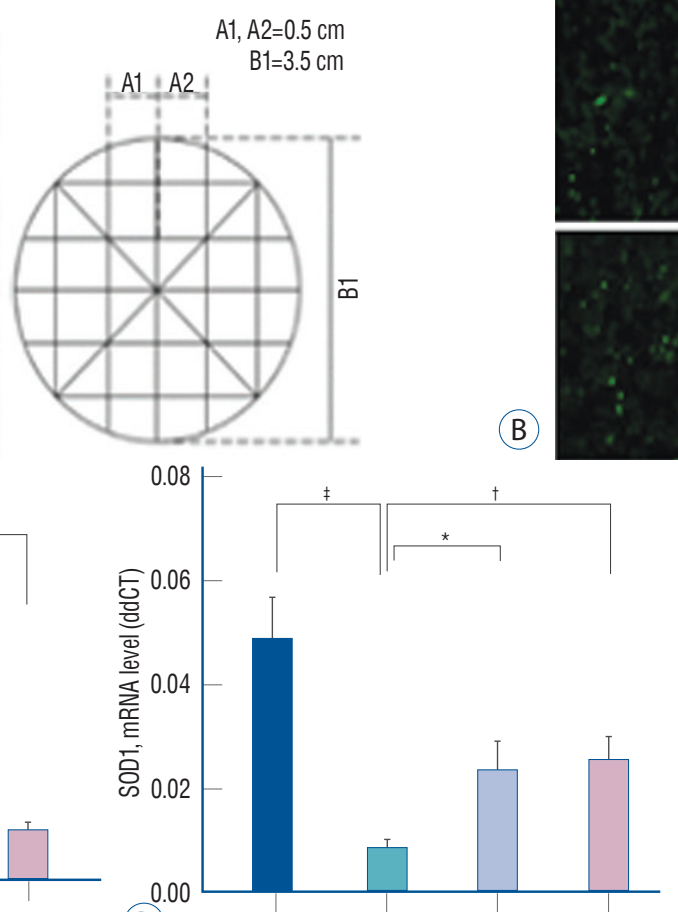

(D)

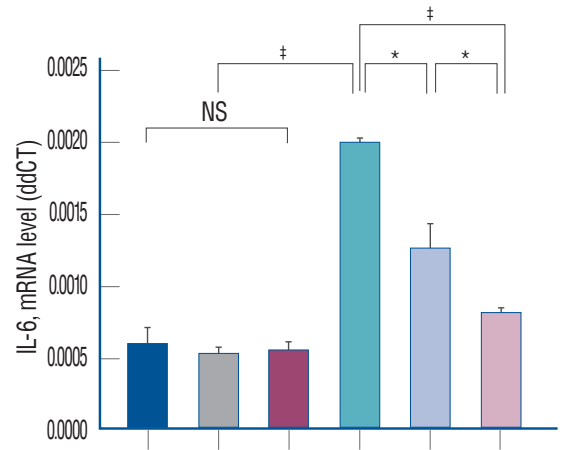

(F)

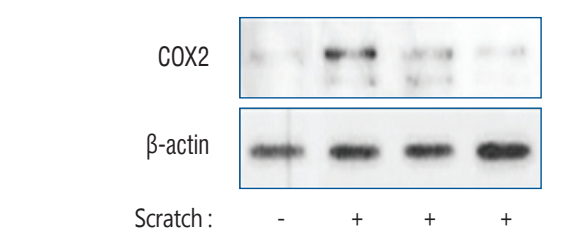

$\mathrm{CeO}_{2} \mathrm{NSs}(1.16 \mathrm{mM})$ :

$\mathrm{CeO}_{2} \mathrm{NRs}(1.16 \mathrm{mM})$ :

(1)

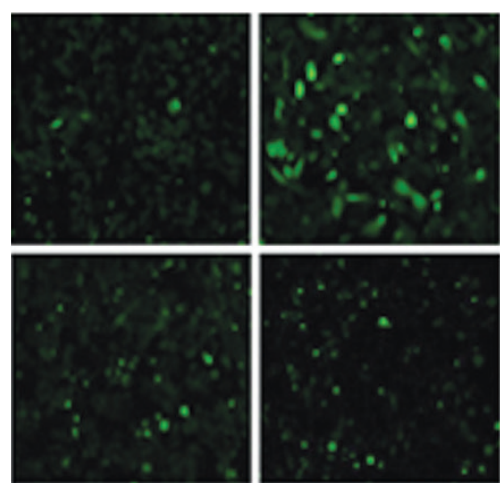

№ TBI+DPBS

$\square$ No TBl+CeO 2 NSs (1.16 mM)

No TBl+CeO ${ }_{2} \mathrm{NRs}(1.16 \mathrm{mM})$

TBI+DPBS

$\mathrm{TBl}+\mathrm{CeO}_{2} \mathrm{NSs}(1.16 \mathrm{mM})$

$\mathrm{TBl}+\mathrm{CeO}_{2} \mathrm{NRs}(1.16 \mathrm{mM})$

Fig. 3. An in vitro model of traumatic brain injury (TBI) induced by static mechanical injury in SH-SY5Y cells (A). Comparison of antioxidant and antiinflammatory effects in an in vitro TBI model using DCFH-DA staining (B $[\times 20]$ and $C)$, and the analysis of mRNA expression of SOD1 and SOD2 (D and E), IL-6 (F), IL-1 $\beta(G)$, and TNF- $\alpha(H)$, using quantitative real-time polymerase chain reaction are shown. Western blot analysis of COX2 expression in a model of cellular TBI (I) and quantification of blots using the relative optical densities of COX2 and $\beta$-actin protein (J). Error bars, mean $\mathrm{SEM}$. ${ }^{*} p<0.05 .{ }^{\dagger} p<0.01$. ${ }^{\ddagger} p<0.001$. LPS : lipopolysaccharides, PMA : phorbol-12-myristate-13-acetate, DPBS : Dulbecco's phosphate-buffered saline, NS : non-specific, TNF-a : tumor necrosis factor-a. 
scavenging activity assay of ceria nanoparticles was performed using a Griess Reagent (Promega, Madison, WI, USA). Briefly, RAW264.7 cells $\left(5.0 \times 10^{5}\right.$ cells/well) in 96-well plate were stimulated by the addition of $1 \mu \mathrm{g} / \mathrm{mL}$ of LPS with or without 1.16 $\mathrm{mM}$ of ceria nanoparticles for 24 hours and 48 hours. Griess reagent was incubated with culture supernatant for $10 \mathrm{~min}$ utes at room temperature followed by measurement of absorbance at $550 \mathrm{~nm}$.

\section{3-(4,5-dimethylthiazol-2-Yl)-2,5-diphenyltetrazo- lium bromide (MTT) assay}

The SH-SY5Y cells $\left(5 \times 10^{5}\right.$ cells/well) were seeded on 96-well culture plates in complete medium overnight, followed by treatment with $1.16 \mathrm{mM}$ of Ceria NRs and Ceria NSs for 24 hours (Fig. 1C). At respective time points, $50 \mu \mathrm{L}$ MTT tetrazolium (3-(4,5-dimethylthiazolyl-2-2,5-diphenyltetrazolium bromide; Sigma-Aldrich-Merck, Saint Louis, MO, USA) solution from the stock $(5 \mathrm{mg} / \mathrm{mL})$ was added and the cells were incubated in a $\mathrm{CO}_{2}$ incubator in the dark for 2 hours. The solution was removed and dissolved using $100 \mu \mathrm{L}$ of DMSO. The absorbance was read at $570 \mathrm{~nm}$ within 30 minutes on a Glo$\operatorname{Max}^{\circledR}$ Discover Microplate Reader (Promega ${ }^{\mathrm{TM}}$, Madison, WI, USA). All experiments were performed in the dark.

\section{In vitro model of TBI}

An in vitro model of TBI was developed by modifying the procedure described previously ${ }^{23)}$. Briefly, six-well confluent plates were manually scratched with a sterile pipette tip using a $6 \times 6$-square grid ( $5 \mathrm{~mm}$ spacing between the lines). More specifically, SH-SY5Y cells were scratched with or without 1.16 $\mathrm{mM}$ of ceria nanoparticles, followed by incubation of the cells as follows : cells without injury (control), cells with a scratch injury, and cells with scratch injuries treated with $1.16 \mathrm{mM}$ of ceria nanoparticles. Following incubation for 24 hours at $37^{\circ} \mathrm{C}$, the medium was removed and washed three times. Intracelluar ROS scavenging activity of ceria nanoparticles was measured as dichloro-dihydro-fluorescein diacetate (DCFH-DA) (Molecular Probes. Inc., Cambridge, MA, USA) fluorescence. Cultured cells were subjected to western blots and quantitative real-time polymerase chain reaction (qRT-PCR), followed by protein extraction and western blotting analysis. ROS production was measured via DCFH-DA staining. The study was performed in accordance with the relevant guidelines.

\section{RNA extraction and quantitative RT-PCR}

Total RNAs were extracted using easy blue and reverse transcribed into cDNA using Maxime RT PreMix Kits (iNtRON Biotechology, Inc., Burlington, MA, USA). qRT-PCR was performed in triplicate for each sample using SYBR Green PCR Kits (applied Biosystems, Norwalk, CT, USA) for 40 cycles with a 3 -step program including 15 seconds of denaturation at $94^{\circ} \mathrm{C}, 30$ seconds of annealing at $55^{\circ} \mathrm{C}$, and 30 seconds of extension at $70^{\circ} \mathrm{C}$. Amplification specificity was assessed via melting curve analysis. Glyeraldehyde-3-phosphate dehydrogenase was used an endogenous control. Expression of cDNA via qRT-PCR was analyzed using the $2^{-\Delta \Delta \mathrm{ct}}$ method. The primer sequences for qRT-PCR are as follows : SOD1, forward 5'-TGAAGAGAGGCATGTTGGAGA-3' and reverse 5'-TGCCCAAGTCATCTGCTTTTT-3'; SOD2, forward 5'-GGAAGCCATCAAACGTGACTT-3' and reverse 5'-GCAGTGGATCCTGATTTGGAC-3'; interleukin (IL)-6, forward 5'-AATTCGGTACATCCTCGACGG-3' and reverse 5'-GGTTGTTTTCTGCCAGTGCC-3'; IL-1 $\beta$, forward 5'-ATGATGGCTTATTACAGTGGCAA-3' and reverse 5'-GTCGGAGATTCGTAGCTGGA-3'; tumor necrosis factor- $\alpha$ (TNF- $\alpha$ ), forward 5'-TGTAGCCCATGTTGTAGCAAACC-3' and reverse 5'-GAGGACCTGGGAGTAGATGAGGTA-3'; actin, forward 5'-GTGCTATCCCTGTACGCCTC-3' and reverse 5'GGCCATCTCTTGCTCGAAGT-3'.

\section{Protein extraction and western blotting analysis}

The protein concentrations were measured using Pierce BCA Protein Assay Kit (Thermo Fisher Scientific Inc., Waltham, MA, USA) after lysis of cells in radio-immunoprecipitation assay buffer supplemented with proteinase inhibitor cocktail. The proteins $(15 \mu \mathrm{g})$ were separated by $10 \%$ sodium dodecyl sulfate-polyacrylamide gel electrophoresis and transferred onto polyvinylidene fluoride membranes. The membranes were blocked with $10 \%$ bovine serum albumin for 1 hour at room temperature and incubated with primary antibodies overnight at $4^{\circ} \mathrm{C}$. The primary antibodies used in this study were anti-COX-2 (sc-7877; 1 : 1000; Abcam, Cambridge, MA, USA) and anti- $\beta$-actin (ab8227; 1 : 1000; Abcam). The blots on the membranes were washed three times for 15 minutes with tris-buffered saline with $0.1 \%$ Tween 20 and then incubated with horseradish peroxidase-linked secondary antibodies. Blots were exposed on an X-ray film for 30 seconds and analyzed by Image J software (Image J $1.49 \mathrm{v}$; National 
Institutes of Health, Bethesda, MD, USA).

\section{Statistical analysis}

Results of the data are expressed as the mean \pm standard error of the mean. Student's t-test or one-way ANOVA with post-hoc Bonferroni correction was performed for all possible pair-wise comparison ${ }^{6)}$. $p$-values $<0.05,<0.01$, and $<0.001$ are represented by ${ }^{*}{ }^{\dagger}$, and ${ }^{*}$, respectively. All statistical analyses were carried out using GraphPad Prism software (v.6.01; GraphPad Software Inc., San Diego, CA, USA).

\section{RESULTS}

The MTT assay results indicated the absence of adverse effects (loss of cell viability) of ceria nanoparticles at a dose of $1.16 \mathrm{nM}$ (Fig. 1C). Also, there was no significant difference in cell viability according to ceria shape. The antioxidant effect of the ceria nanoparticles was determined using Superoxide anion assays. Ceria NRs and Ceria NSs reduced the generation of superoxide anion compared with Dulbecco's phosphatebuffered saline after stimulation of LPS-primed U937 cells with PMA (Fig. 2A). The release of nitrite, which is the predominant metabolite of $\mathrm{NO}$, into the culture medium of RAW264.7 cells under LPS stimulation was detected using a Griess reagent. When cells were treated with a combination of ceria nanoparticles and $1 \mu \mathrm{g} / \mathrm{mL}$ of LPS, the release of nitrite at 24 and 48 hours was significantly reduced compared with the level in the group treated with LPS only (Fig. 2B).

Following TBI-induced changes in SH-SY5Y cells, the DCFH-DA assay was performed to determine the differences in total intracellular ROS under confocal laser scanning microscopy in the different treatment groups (Fig. 3A-C). Ceria NRs and Ceria NSs significantly reduced the ROS levels inside cells compared with the control group. Reduced total ROS level showed similar changes in the superoxide anion assay. Based on these results, the mRNA expression of SODs was quantified using qRT-PCR. Ceria nanoparticles reduced the expression of SOD1 and SOD2 in SH-SY5Y cells compared with control (Fig. 3D and E). Ceria NRs and Ceria NSs did not alter the decrease in the mRNA expression of SOD1 and SOD2. In addition, Ceria NRs had lower levels of IL-6, IL-1 $\beta$, and TNF- $\alpha$ than Ceria NSs (Fig. 3F-H). Ceria nanoparticles also decreased the COX2 level, which is responsible for induc- ing inflammation, in the cellular TBI model (Fig. 3I and J). Ceria NRs $(0.438 \pm 0.105)$ showed better anti-inflammatory activity than ceria NSs $(1.321 \pm 0.139 ; p=0.025)$.

\section{DISCUSSION}

Effective delivery of the nanoparticles into the injured brain is a challenge due to the presence of blood-brain barrier (BBB). $\mathrm{BBB}$ is composed of vascular endothelial cells, neurons, and glial cells. It stabilizes the central nervous system by preventing the entry of exogenous compounds and blood-borne substances from brain parenchyma ${ }^{1,7)}$. TBI loosens the tight junctions, and thereby increases the permeability of BBB, which leads to an influx of inflammasomes into the brain. This phenomenon not only occurs immediately after TBI, but also after a few days. Baldwin et al. ${ }^{3)}$ showed a biphasic opening of the BBB following severe TBI, suggesting the need for adequate treatment of secondary brain injury similar to the treatment immediately post-TBI. Oxidative stress due to an imbalance in pro-oxidant-antioxidant levels plays a vital role in the pathophysiology of secondary brain injury ${ }^{17)}$. An increase in ROS production during the oxidation of proteins and nucleic acids can lead to cellular membrane disruption, which increases the release of neuroinflammatory markers such as IL6 , COX2, and TNF- $\alpha^{9)}$. In addition, increased oxidative stress and inflammatory response resulted in structural changes of the brain, even in mild TBI. Dall'Acqua et al. ${ }^{4)}$ reported a functional hypoconnectivity in the early phase following mild TBI. The structural and functional network was impaired 1 year after trauma, despite partial normalization. Accordingly, effective treatment of TBI requires an increase in the antioxidant effects and reduction in acute and chronic inflammation in the brain by stabilizing the BBB long term.

Pharmacological studies investigated the role of glycol-conjugated SOD, tirilazad mesylate, and dexanabinol in effectively decreasing oxidative stress and neuroinflammation. Hamm et al. ${ }^{5)}$ reported that polyethylene glycol-conjugated SOD reduced the neurological damage via free radical scavenging effect and improved cerebral blood flow. Treatment with tirilazad mesylate reduced the mortality rate of male patients with severe $\mathrm{TBI}^{12}$. However, dexanabinol did not show therapeutic effects based on the extended Glasgow outcome scale at 6 months compared with the placebo group ${ }^{11)}$. Since the 2010 s, 
ceria nanoparticles have been used to treat brain injury. Ceria nanoparticles exhibit antioxidant and anti-inflammatory effects via rapid $\mathrm{Ce}^{3+} / \mathrm{Ce}^{4+}$ cycling in the catalytic pathway. Such redox properties depend on the shape and size of the nanoparticles. Compared with Ceria NSs, Ceria NRs were less stable and contained high-energy reactive crystal planes. Ceria NRs provided higher oxygen storage capacity and catalytic activity for CO oxidation ${ }^{22,24)}$. In addition, Ceria NRs exhibited a higher $\mathrm{Ce}^{3+} / \mathrm{Ce}^{4+}$ molar ratio on the surface (0.40) than Ceria NSs (0.27). Accordingly, it is possible that Ceria NRs manifest a better therapeutic effect than Ceria NSs. In our study, the mRNA expression of SOD1 and SOD2, and protein levels of COX2 were decreased in the group treated with ceria nanoparticles. However, Ceria NRs and Ceria NSs similarly decreased the mRNA expression of SOD1 and SOD2, although Ceria NRs showed better anti-inflammatory activity than Ceria NSs. The relatively high dosage of nanoparticles used in this experiment may affect the outcomes. Optimal therapeutic effects were found in in vitro models of mild TBI with $10 \mathrm{nM}$ of ceria nanoparticles compared with $1 \mathrm{nM}$ and $100 \mathrm{nM}$. Although the dosage of ceria nanoparticles was based on a previous report ${ }^{6}$, , an appropriate dosage has yet to be determined by testing the toxicity of various concentrations. Further efforts are needed to enhance the therapeutic effects of nanoparticles via changes in structural properties or mixing ceria with other substances, and not based on relative changes in nanoparticle shape. Nance et al. ${ }^{14)}$ reported that neutral dendrimers are transported efficiently to the injured brain and localized in the glial cells. In addition, manganese or cobalt may enhance the catalytic effects. Thus, a follow-up study to investigate the in vivo and in vitro effects is needed under various conditions.

The study limitations are as follows. First, it is difficult to reproduce secondary brain injury after TBI via in vitro study. In addition, we compared only the antioxidant and anti-inflammatory effects of Ceria NRs and Ceria NSs. Accordingly, our findings are limited because actual regeneration of damaged brain cells and recovery of the neurological function cannot be achieved. Thus, the effects of Ceria NRs and Ceria NSs should be identified in different types of neurons in the brain via follow-up studies. Second, the distribution of Ceria NRs in the brain should be investigated using an in vivo model of TBI, although a single injection of ceria nanoparticles was distributed well in the brain ${ }^{2)}$. Third, the degree of TBI dam- age may affect the effects of ceria nanoparticles. This study represents a proof-of-concept study to support our hypothesis. Accordingly, various degrees of stretch injury using the cell injury controller are needed to further corroborate our results.

\section{CONCLUSION}

Ceria nanoparticles exhibited antioxidant and anti-inflammation effects in in vitro models of TBI. No significant difference was detected between the antioxidant activities of Ceria NRs and Ceria NSs, although Ceria NRs showed a pronounced anti-inflammatory effect.

\section{CONFLICTS OF INTEREST}

No potential conflict of interest relevant to this article was reported.

\section{INFORMED CONSENT}

This type of study does not require informed consent.

\section{AUTHOR CONTRIBUTIONS}

\author{
Conceptualization : JPJ, HY \\ Data curation : DHY, NMT \\ Formal analysis : $\mathrm{HJ}$ \\ Funding acquisition : JPJ \\ Methodology : DHY, NMT, HJ \\ Project administration : JPJ \\ Visualization: DHY, NMT \\ Writing - original draft : JPJ, HJ \\ Writing - review \& editing: JPJ, HY
}

\section{ORCID}

Dong Hyuk Youn https://orcid.org/0000-0003-2259-1844

Harry Jung https://orcid.org/0000-0002-7691-8403

Ngoc Minh Tran https://orcid.org/0000-0003-2116-6707 
Jin Pyeong Jeon ～https://orcid.org/0000-0001-8543-6855 Hyojong Yoo https://orcid.org/0000-0002-4932-3885

\section{- Acknowledgements}

This research was supported by the "Real National R\&D Challenge Program" grant funded by the Korea Institute of Human Resources Development in Science \& Technology (NRF-4R210101523S000100), Hallym University Research Fund, and the National Research Foundation of Korea funded by the Ministry of Education (2020R111A3070726).

We thank Youngmi Kim for assistance with the experimental procedure and the overall study.

\section{References}

1. Alam Bony B, Kievit FM : A role for nanoparticles in treating traumatic brain injury. Pharmaceutics $11:$ 473, 2019

2. Bailey ZS, Nilson E, Bates JA, Oyalowo A, Hockey KS, Sajja VSSS, et al. : Cerium oxide nanoparticles improve outcome after in vitro and in vivo mild traumatic brain injury. J Neurotrauma 37 : 1452-1462, 2020

3. Baldwin SA, Fugaccia I, Brown DR, Brown LV, Scheff SW : Blood-brain barrier breach following cortical contusion in the rat. J Neurosurg 85 : 476-481, 1996

4. Dall'Acqua P, Johannes S, Mica L, Simmen HP, Glaab R, Fandino J, et al. : Functional and structural network recovery after mild traumatic brain injury: a 1-year longitudinal study. Front Hum Neurosci 11 : 280, 2017

5. Hamm RJ, Temple MD, Pike BR, Ellis EF : The effect of postinjury administration of polyethylene glycol-conjugated superoxide dismutase (pegorgotein, Dismutec) or lidocaine on behavioral function following fluid-percussion brain injury in rats. J Neurotrauma 13 : 325-332, 1996

6. Jeong HG, Cha BG, Kang DW, Kim DY, Ki SK, Kim SI, et al. : Ceria nanoparticles synthesized with aminocaproic acid for the treatment of subarachnoid hemorrhage. Stroke 49 : 3030-3038, 2018.

7. Kadry H, Noorani B, Cucullo L : A blood-brain barrier overview on structure, function, impairment, and biomarkers of integrity. Fluids Barriers CNS $17: 69,2020$

8. Kaur $\mathrm{P}$, Sharma $\mathrm{S}$ : Recent advances in pathophysiology of traumatic brain injury. Curr Neuropharmacol 16 : 1224-1238, 2018

9. Khatri N, Thakur M, Pareek V, Kumar S, Sharma S, Datusalia AK : Oxidative stress: major threat in traumatic brain injury. CNS Neurol Disord Drug Targets $17: 689-695,2018$
10. Kim CK, Kim T, Choi IY, Soh M, Kim D, Kim YJ, et al. : Ceria nanoparticles that can protect against ischemic stroke. Angew Chem Int Ed Engl 51 : 11039-11043, 2012

11. Maas Al, Murray G, Henney H 3rd, Kassem N, Legrand V, Mangelus $M$, et al. : Efficacy and safety of dexanabinol in severe traumatic brain injury: results of a phase III randomised, placebo-controlled, clinical trial. Lancet Neurol 5 : 38-45, 2006

12. Marshall LF, Maas AI, Marshall SB, Bricolo A, Fearnside M, lannotti F, et al. : A multicenter trial on the efficacy of using tirilazad mesylate in cases of head injury. J Neurosurg 89 : 519-525, 1998

13. Najem D, Rennie K, Ribecco-Lutkiewicz M, Ly D, Haukenfrers J, Liu Q, et al. : Traumatic brain injury: classification, models, and markers. Biochem Cell Biol 96 : 391-406, 2018

14. Nance E, Zhang F, Mishra MK, Zhang Z, Kambhampati SP, Kannan RM, et al. : Nanoscale effects in dendrimer-mediated targeting of neuroinflammation. Biomaterials 101 : 96-107, 2016

15. Nelson BC, Johnson ME, Walker ML, Riley KR, Sims CM : Antioxidant cerium oxide nanoparticles in biology and medicine. Antioxidants (Basel) 5 : 15, 2016

16. Pan A, Zhu T, Wu HB, Lou XW : Template-free synthesis of hierarchical vanadium-glycolate hollow microspheres and their conversion to V205 with improved lithium storage capability. Chemistry 19 : 494-500, 2013

17. Rodríguez-Rodríguez A, Egea-Guerrero JJ, Murillo-Cabezas F, Carrillo-Vico A : Oxidative stress in traumatic brain injury. Curr Med Chem 21 : 1201-1211, 2014

18. Rzigalinski BA, Meehan K, Davis RM, Xu Y, Miles WC, Cohen CA : Radical nanomedicine. Nanomedicine (Lond) 1 : 399-412, 2006

19. Torrente-Murciano L, Gilbank A, Puertolas B, Garcia T, Solsona B, Chadwick D : Shape-dependency activity of nanostructured $\mathrm{CeO} 2$ in the total oxidation of polycyclic aromatic hydrocarbons. Appl Catal B 132-133: 116-122, 2013

20. Youn DH, Tran NM, Kim BJ, Kim Y, Jeon JP, Yoo H : Shape effect of cerium oxide nanoparticles on mild traumatic brain injury. Sci Rep 11 : 15571, 2021

21. Zavvari F, Nahavandi A, Shahbazi A : Neuroprotective effects of cerium oxide nanoparticles on experimental stress-induced depression in male rats. J Chem Neuroanat 106 : 101799, 2020

22. Zhang M, Li J, Li H, Li Y, Shen W : Morphology-dependent redox and catalytic properties of $\mathrm{CeO} 2$ nanostructures: nanowires, nanorods and nanoparticles. Catal Today 148 : 179-183, 2009

23. Zhao Y, Luo P, Guo Q, Li S, Zhang L, Zhao M, et al. : Interactions between SIRT1 and MAPK/ERK regulate neuronal apoptosis induced by traumatic brain injury in vitro and in vivo. Exp Neurol 237 : 489-498, 2012

24. Zhou K, Li Y : Catalysis based on nanocrystals with well-defined facets. Angew Chem Int Ed Engl 51 : 602-613, 2012 